\title{
Citations
}

\section{The Big Picture}

Imaging mass spectrometry (MS) of tissue sections examines the spatial distribution of ions whose identities are confirmed in additional MS steps. For small molecules, on-tissue tandem MS (MS/MS) is possible, but protein identification poses a more substantial challenge. One approach is to remove a tissue sample for homogenization and digestion; although this is a time-tested proteomic methodology, it destroys the sample. Alternatively, an adjacent tissue section can be used for in situ proteolytic digestion, followed by on-tissue MS/MS. However, this method yields fewer tryptic peptides, and attempts to improve digestion efficiency tend to disrupt the tissue's structural integrity, compromising spatial resolution. Now, an article in Analytical Chemistry from Harris et al. suggests a way to combine the advantages of in situ digestion and in-tube peptide extraction. In the new method, a trypsin-impregnated hydrogel disc is placed on a tissue section and incubated to allow on-tissue digestion. The gel is then removed and subjected to extractions with organic and aqueous solvents, and extracts are analyzed by MALDI (matrix-assisted laser desorption ionization) MS and liquid chromatography-MS/MS (LC-MS/ MS). Experiments with rat brain showed the hydrogel-based procedure identified the majority of the proteins found in directly extracted tissue punches, including high-molecular-weight proteins that typically prove elusive in on-tissue MS/MS. Most appealing is the ease with which the hydrogel-based method fits into a full imaging workflow. To demonstrate this, Harris et al. took a rat brain section and subjected it to imaging MS and on-tissue MS/ MS for analysis of lipid species, taking care to use laser energies below the range that would cause tissue ablation. The authors then performed hydrogel-based digestion and LC-MS/MS, identifying 96 proteins, including many of high molecular weight. Thanks to

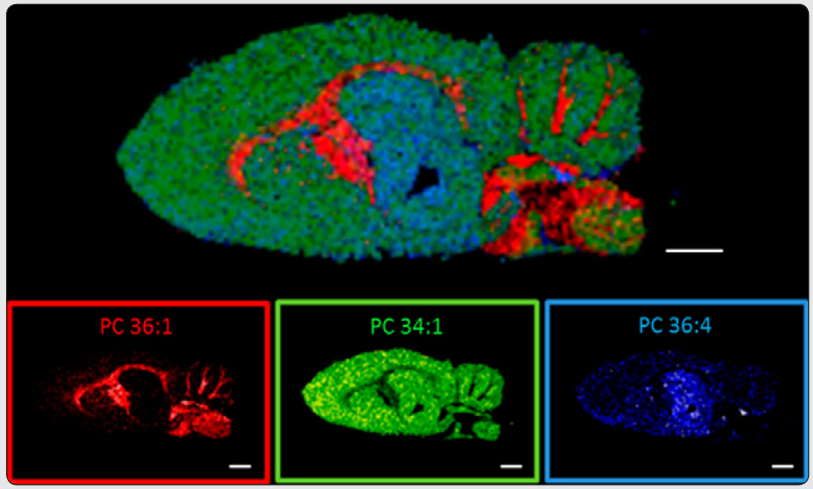

Imaging MS of lipid species in a rat brain slice prior to hydrogel-mediated protein digestion and extraction. Source: Analytical Chemistry.

the largely nondestructive nature of the procedure, the section was still available for further analysis, and Harris et al. showed that they could then use histological staining to identify myelination boundaries and other brain regions, even if some detail had been lost. In future work, the authors hope to construct smaller hydrogel discs for greater resolution, improve digestion and extraction efficiency, and develop new staining protocols that minimize cell loss during washing steps. Together, these innovations should help render protein distribution in tissue sections in arresting detail.

Harris et al. localized in situ hydrogel-mediated protein digestion and extraction technique for on-tissue analysis. Anal Chem. [Epub ahead of print, February 12, 2013; doi:10.1021/ ac3031493].

\section{Fast Forward}

Forward genetics has been a prime source of gene function insights for decades. The trickiest step, identifying the causal mutation, has been revolutionized by whole genome sequencing (WGS) in isogenic model invertebrates with relatively small genomes such as the roundworm and the fruit fly. WGS is less economical in zebrafish, whose genome is about an order of magnitude larger. Adding to the expense is the genome's highly polymorphic nature, meaning multiple individuals must be sequenced to sort the causative mutation from bystander polymorphisms. Even so, the old school alternative of linkage mapping with microsatellite markers is unappealing because of its laboriousness and limited resolution. Instead, Miller et al., writing in Genome Research, propose an RNA-seq-based mapping procedure. Like WGS, RNA-seq provides nucleotide level resolution; however, because sequencing is limited to expressed genes, high coverage can be obtained at substantially lower cost. In their pipeline, Miller et al. prepared RNA from pools of zebrafish embryos with and without the mutant phenotype, created libraries for next-generation sequencing, and then used high-quality SNPs as markers for linkage analysis. Once a linked region was found, all SNPs were extracted and then filtered to remove those previously observed in wild-type zebrafish, focusing on candidate SNPs corresponding to missense or nonsense mutations. Splicing defects, a class of mutations more difficult to uncover by WGS, could be picked up by visual comparison with annotated intron/ exon boundaries. In tests, Miller et al. found that pools of 20-40 embryos were sufficient to pinpoint a handful of candidate SNPs as potentially causal. The authors provide their bioinformatics pipeline for download at www.RNAmapper.org and suggest that the overall technique should be portable to other vertebrate model organisms with similarly sized genomes (e.g., Xenopus, mouse, rat). Aside from the key advantages of requiring less sequencing and allowing easier detection of splicing mutations, Miller et al. point out that their workflow may give direct evidence of mutations causing changes in transcript expression levels. At present, the time-efficient RNA-seq technique offers cost reductions of $67 \%$ to $83 \%$ compared with WGS, with potential for extra savings via additional multiplexing.

Miller et al. RNA-seq-based mapping and candidate identification of mutations from forward genetic screens. Genome Res. 2013 Feb 4 . [Epubahead of print, February 4, 2013; doi:10.1101/gr.147322.112].

Selected and written by Nijsje Dorman, Ph.D. 四A

BioTechniques 54: 181 (April 2013) doi 10.2144/000114005 